\title{
PREVENTIVE AND REGENERATIVE RESPONSES TO 1- ISOTHIOCYANATO-4-(METHYLSULFINYL) BUTANE IN ANNULAR PUNCTURE - INDUCED MODEL OF RABBIT INTERVERTEBRAL DISC DEGENERATION 1,20gunlade B, ${ }^{1 *}$ Adelakun SA, ${ }^{3}$ Fidelis OP, ${ }^{3}$ Owolabi FM
}

\author{
${ }^{1}$ Anatomy Department, Federal University of Technology Akure, Ondo State. \\ ${ }^{2}$ Anatomy Department, College of Medicine, University of Lagos. \\ ${ }^{3}$ Biomedical Technology Department, Federal University of Technology Akure, Ondo State.
}

Correspondence to Adelakun Sunday A. Department of Human Anatomy, School of Health and Health Technology, Federal University of Technology, Akure, Ondo State, Nigeria.

E-mail address: saadelakun@futa.edu.ng

\begin{abstract}
This study aimed at investigating the preventive and rejuvenating capacity of 1-isothiocyanato-4(methylsulfinyl) butanein annular punctured intervertebral disc degeneration in rabbit model.Twentyfive New Zealand white rabbits (1.5 to $3.0 \mathrm{~kg}$ each) underwent annular puncture.Group 1 received $50 \mathrm{mg} / \mathrm{kg}$ of phosphate-buffered saline orally for 8 weeks (non-punctured group); group 2 received $50 \mathrm{mg} / \mathrm{kg}$ phosphate-buffered salinesolution immediately after puncture for 8 weeks (model saline group I); group 3 received $50 \mathrm{mg} / \mathrm{kg}$ phosphate-buffered salinesolution after 8 weeks of puncture for another 8 weeks (model saline group II); group 4 received the oral administration of $450 \mathrm{mg} / \mathrm{kg}$ of 1-isothiocyanato-4(methylsulfinyl) Butane solution immediately after puncture for 8 weeks; group 5 received the oral administration of $450 \mathrm{mg} / \mathrm{kg}$ of 1-isothiocyanato-4-(methylsulfinyl) Butane solution after 8 weeks of puncture for another 8 weeks.Serial X-rays were performed at 0, 8 and 16weeks for the disc height. Upon sacrifice, the whole spinal column and discs were extracted and analyzed for various histological staining techniques (H\&E and HVG), biochemical and immunohistochemical analysis. There wasdecreased in disc height in the $\mathrm{X}$ - ray and was significantly prevented and regenerated after the administration of 1-isothiocyanato-4-(methylsulfinyl) Butane solution. The histological grade, collagen type 1 and 2, aggrecan, and matrix metalloprotease-13 mRNA expression and histological analysiswere definitive in the degeneration processes thereby affirming the X-ray data. This study therefore ascertain that 1-isothiocyanato-4-(methylsulfinyl) Butane had anabolic effects on degenerated disc and could provide valuable information for consideration in clinical trials.
\end{abstract}

Keywords: Low back pain, intervertebral disc degeneration, 1-isothiocyanato-4-(methylsulfinyl) Butane, histology.

\section{INTRODUCTION}

The most frequent cause of physical disability in both developed and developing nations is musculoskeletal disorders (Murray and Lopez 1997; Lopez et al., 2006). Among the major musculoskeletal conditions that could lead to disability in every individual, Low Back Pain (LBP) is the most prevalent and the most common cause of disability in developed nations (Woolf and Pfleger, 2003). LBP as a result of disc degeneration costs billions of dollars in medical expenditures each year (Childs et al., 2004) and this economic burden in a continent such as Africa will be enormous, where health care funds are already directed toward epidemics such as 
HIV and AIDS. Socioeconomic constraints in Africa are therefore a predisposing factor to higher prevalence of many diseases and disabilities (Lopez et al., 2006).

Intervertebral disc degeneration (IVDD) has been revealed to be more common in patients with LBP than in asymptomatic individuals (Urban and Roberts, 2003) that are affected by multiple occupational backgrounds and genetic predispositions (Urban and Roberts, 2003; Podichetty, 2007).

Among all the available experimental animal models procedure, the multiple annular needle puncture to the IVD is the most simple, reliable, reproducible, minimally invasive and standardized way to evaluate intervertebral disc degeneration (Masubaet al., 2005; Rousseau et al., 2007). This method can cause a slow, cumulative degeneration with anatomical, pathological, biochemical and molecular changes similar to those observed in human disc degeneration (Masubaet al., 2005).

Several methods are been used to evaluate experimental disc degeneration which have proved to be standardized by using radiographs, tomography, magnetic resonance imaging, and biochemical, histological and molecular analysis (Lotz 2004; Elliott and Sarver 2004;Vaille et al., 2009). Among all the standardized method, histological analysis could be considered a gold standard for experimental disc degeneration evaluation and grading. It has been reported that consumption of cruciferous vegetables such as broccoli, kale and cabbage lower risk of cancer and age related diseases (Zhao et al., 2016). These beneficial effects have been attributed to isothiocyanates (ICT) derived from glucosinolate precursors that are specifically numerous in cruciferous vegetables (DinkovaKostova 2013). 1-isothiocyanato-4(methylsulfinyl) Butane (SFN), is the most naturally interesting and studied ICTs present in crucifers (Zhang et al., 1992). It has been reported that SFN can promote elimination and detoxification of aflatoxin (Kensleret al., 2005), acetaldehyde (Ushida and Talalay 2013), methylmercury (Toyama et al., 2011), acrolein (Egneret al., 2014), benzene (Kensleret al., 2013), crotonaldehyde (Kensleret al., 2013) and free radicals (Gaona-Gaonaet al., 2011) through the Nrf2-mediated mechanism. It is also capable of exerting chemoprotective effects against cancers via"blocking" and "suppressing" effects (Talalayet al., 1995; Lenziet al., 2014) and against chronic diseases such as liver failure (Sayed et al., 2014), cerebral nerve diseases (Shiraiet al., 2012; Shiraiet al., 2015), and maculardegeneration (Gao and Talalay 2004) in experimental animal models.

\section{MATERIALS AND METHOD}

\section{Drug source}

Pure 1-isothiocyanato-4-(methylsulfinyl) Butane was obtained from Sigma- Aldrich, MO, USA and was dissolved in phosphate-buffered saline.

\section{Surgical technique}

All animal handling and surgical procedures were conducted in accordance with the ethical approval from the Health Research Ethics Committee, College of Medicine of the University of Lagos.

Twenty-five (25) New Zealand White rabbits (weighing about $1.5-3.0 \mathrm{~kg}$ ) were used with institutional animal care committee's approval. The surgical technique was performed using modified Young-Joon(2013) procedure. Briefly, each rabbit was anesthetized with intramuscular injection of xylazine $(5 \mathrm{mg} / \mathrm{kg}$ ) and ketamine (35 $\mathrm{mg} / \mathrm{kg}$ ), and the fur was shaved from the mid back and right flank. After anesthesia, a lateral plain X-ray was obtained to establish the preinjection baseline height of the IVDs. The rabbit was then placed in the lateral oblique prone position, and the injection field was sterilized with an alcohol sponge. Initially, the L5-L6 disc was identified through manual palpation of the interspinous space from the mid back and pelvic rim. After confirmation of the exact level, a 21gauge angiography needle was inserted 3-4 cm ventrally from the midline into the disc space. After brief confirmation of the needle position in the center of the disc space, the needle was held in the disc space for 30 seconds. Before removal, 
the needle was rotated 360 degrees. In each rabbit, each of three discs (L3-L4, L4-L5 and L5L6) was punctured. The L1-L2 and L2-L3 levels were designated as the non-punctured, internal controls. For each level, all procedures for identification and puncture were performed within a calculated time. Special care was taken to minimize contact with the periosteal tissues of the vertebrae because this could cause hypertrophy of the soft tissues and bony structures around the discs. Rabbits were monitored for neurological symptoms. The rabbits were placed in their cages after observation for recovery.

The rabbits were divided into 5 animals per group:

1. Group 1 received $50 \mathrm{mg} / \mathrm{kg}$ of phosphate-buffered saline orally for 8 weeks (non-punctured group)

2. Group 2 received $50 \mathrm{mg} / \mathrm{kg}$ phosphatebuffered salinesolution immediately after puncture for 8 weeks (model saline group I)

3. Group 3 received $50 \mathrm{mg} / \mathrm{kg}$ phosphatebuffered salinesolution after 8 weeks of puncture for another 8 weeks (model saline group II)

4. Group 4 received the oral administration of $450 \mathrm{mg} / \mathrm{kg}$ of 1-isothiocyanato-4(methylsulfinyl) Butane solution immediately after puncture for 8 weeks

5. Group 5 received the oral administration of $450 \mathrm{mg} / \mathrm{kg}$ of 1-isothiocyanato-4(methylsulfinyl) Butane solution after 8 weeks of puncture for another 8 weeks

Lateral X-ray of the lumbar spine was taken before and after the experiment to measure IVD height. After the experiments, rabbits were euthanatized and the IVDs were assessed. As an internal control, the non-punctured discs (L1/2 and $\mathrm{L} 2 / 3$ ) were also assessed.

At the end of the experiment, the animals were sacrificed with intramuscular injection of ketamine $(25.0 \mathrm{mg} / \mathrm{kg})$ followed by intravenous injection of sodium pentobarbital $(1.2 \mathrm{~g} / \mathrm{kg})$.

\section{Radiologic analysis}

The rabbits were anesthetized and a lateral plane radiograph of the lumbar spine was taken with a radiograph machine (collimator-to film distance, $50 \mathrm{~cm}$; exposure, $5 \mathrm{~m}$ as; penetration power, $44 \mathrm{kVp}$ ). During the radiographs, special care was taken to minimize axial rotation of the disc space by holding rabbits in the lateral decubitus position while ensuring the X-ray beam is maintained straight. The lateral film was confirmed to be straight by checking for overlap of both transverse processes of the spine. The $\mathrm{X}$-ray was repeated if a straight film is not obtained. To decrease the error from beam divergence, the beam was centred at $4 \mathrm{~cm}$ from the iliac crest. In addition, each rabbit was treated with a consistent amount of anaesthesia in order to provide a similar degree of muscle relaxation to minimize differences in disc height. The IVD height was expressed as the disc height index (DHI) where (DHI = IVD height/adjacent vertebral disc height) (Masuda et al., 2006). Change in the DHI of injected discs was expressed as percentage DHI (\% DHI) and normalized to the measured preoperative IVD height: $\%$ DHI = (postoperative DHI/preoperative DHI) $\times 100$ (Masuda et al., 2006).

\section{Biochemical analysis}

Rabbits were randomly chosen for real time quantitative polymerase chain reaction (PCR) using Young-Joon (2013) procedure. The L3/L4 and L4/L5 levels were extracted for the punctured disc while L1/L2 disc was extracted as a non-punctured control. From each disc, the nucleus pulposus (NP) was carefully removed from annulus fibrosus(AF) and stored separately. The tissues were immediately placed into liquid nitrogen and frozen at $-80^{\circ} \mathrm{C}$ in preparation for PCR analysis. Genes were selected from the representative forms related to the ECM component (collagen type 1 and 2, aggrecan) and catabolic enzymes (matrix metalloprotease13, MMP-13). The frozen NP samples were homogenized using Mini-Beadbeater; Bio Spec, Bartlesville, OK, USA, in $1 \mathrm{~mL}$ Trizol reagent (Invitrogen, Carlsbad, CA, USA). RNA was extracted in accordance with the manufacturer's instructions. The primers for the rabbit-specific genes were designed in accordance with 
published sequences in Young-Joon, (2013) procedure. Gene expression was analysed by RT-PCR using ABI PRISM 9700 (Applied Biosystems, Foster City, CA, USA). Gene expression levels were calibrated using a constitutively expressed housekeeping gene, glyceraldehyde phosphate dehydrogenase (GADPH). A positive standard curve for each primer was obtained using serially diluted CDNA sample mixture. Quantifications of gene expression were calculated (comparative $\mathrm{Ct}$ method) using standard curves and normalized to GAPDH in each sample, and then the expression of treated discs was normalized to control discs.

The sequences were as follows: aggrecan ( $5^{\prime}$ GCTACGGAGACAAG GATGAGTTC $3^{\prime}$ and $5^{\prime}$ CGTAAAAGACCTCACCCTCCAT 3'), MMP-13 (5' TGCCCCTCCTCAACAGTAAC 3 ' and 5'GAGCCCGCTGCATTCTTCTT3'), collagen type II :(5' TCAGGAATTTGGTGTGGACATA-3'and 5'CCGGACTGTGAGGTTAGGATAG 3'), Type I collagen (5' -GGGCAAGACAGTCATCGAATA-3' and 5' GATTGGGATGGAGGGAGTTTA- 3') GAPDH (5' AAGGCCATCACCATCTTCCA 3' and 5' GGATGCGTTGCTGACAATCT 3'). Histomorphological and organization of collagen fibres analyses

Two rabbits were selected randomly before surgery and at 4 weeks after surgery for histological evaluation. The intact specimens, including the annulus fibrosus, the nucleus pulposus (both endplates) and the adjacent vertebral body bone, were be fixed.

The punctured discs and the control discs were harvested for histologic analyses. An electric saw was be used to cut each disc together with the adjacent vertebral body. Tissues were fixed with $10 \%$ neutral buffered formalin for 48 hours, decalcified in decalcification solution (National Diagnostics, Atlanta, GA, USA) for 3 days and processed for paraffin sectioning. Blocks embedded in paraffin were cut into mid-sagittal sections ( $4 \mu \mathrm{m}$ in thickness) with a microtome. The sections were stained with Haematoxylin and Eosin (H\&E) and Haematoxylin van Gieson stain. They were analyzed under a light microscope (Nikon Eclipse E800; Nikon, Melville, NY,
USA) at magnifications ranging from $40 \times$ to 200x.

The degree of IDD were assessed by a histological grading scale (Masuda et al., 2005), with scores ranging from grade 4 (normal) to grade 12 (severely degenerated). This grading scale is based on degenerative changes in 4 regions: the annulus fibrosus, the border between the annulus fibrosus and the nucleus pulposus, the parenchyma of the nucleus pulposus, and the matrix of the nucleus pulposus.

\section{Expression levels of protein (immunohistochemical analysis)}

The expression levels of Bax protein were determined using Le Maitre et al. (2007) protocol. Formalin-fixed, paraffin-embedded 3 $\mu \mathrm{m}$ thick sections were deparaffinized and rehydrated. Endogenous peroxidase activity was blocked by incubating the sections with $3 \% \mathrm{H}_{2} \mathrm{O}_{2}$ for $10 \mathrm{~min}$ followed by digestion with $0.01 \%$ protease $\mathrm{K}$ for $10 \mathrm{~min}$. Non-specific binding sites were blocked by incubation with confining liquid for $10 \mathrm{~min}$ after which the sections were incubated with rat polyclonal antibody to Bax (Cell Signaling Inc., Danvers, MA) at $4{ }^{\circ} \mathrm{C}$ for 12 h. After thorough washing, the sections were incubated with biotinylated goat anti-rabbit IgG at $4{ }^{\circ} \mathrm{C}$ for $60 \mathrm{~min}$ and then in Streptavidin-HRP for $10 \mathrm{~min}$. The final color reaction was developed by incubation with the chromogenic substrate $3,3^{\prime}$-diaminobenzidine $(0.5 \mathrm{mg} / \mathrm{mL}$ in Tris). The sections were counterstained with haematoxylin and mounted for examination with an O-max microscope coupled to Image J software (National Institutes of Health, USA).

\section{Statistical analysis}

Data were analysed using Statistical Package for the Social Sciences version 24.0 (SPSS Inc.; Chicago U.S.A.). Longitudinal X-ray data were analyzed using two-way analysis of variance (ANOVA) followed by multiple comparison using Bonferroni method. Quantitative real time-PCR data were analyzed using student t-test. Data were presented as mean \pm standard error of 
mean. The level of significant was considered at $p<0.05$.

\section{RESULTS}

\section{RADIOLOGICAL ANALYSIS}

Immediately after puncture, the model saline group (group 2) showed a significant narrowing of disc height when compared with that of the non-punctured group (group 1) (approximately $25.0 \%$ decrease compared with the baseline percentage disc height index (DHI), $\mathrm{P}<0.05)$ as shown in Figure 1. There were no significant differences in the percentage DHI among the treated groups that received phosphate buffered saline immediately and after 8 weeks of puncture.

\section{HISTOLOGICAL ANALYSIS}

Photomicrograph of the histological section for groups 4 and 5 revealed that the administration of 1-isothiocyanato-4-(methylsulfinyl) Butane solution preventsand regenerates the distortions in the cytoarchitecture and histomorphology of the IVD compared to that of the model saline group (group 2). The observation of the nonpunctured group (group 1) showed normal IVD morphology. However, model saline groups showed structural disorganization resembling disc degeneration - narrowingof the disc height in which most of the NP contents have been lost and collapse.

\section{HISTOLOGICAL GRADING SCORES}

The histomorphological finding was carried out using a previously described histological grading score for the degeneration of IVD (Boos et al., 2002). The scoring grade ranges from 4 (normal) to 12 (severely degenerated), depending on the level of degeneration. In this study, there were no significant differences in the histological scores among the treated groups that received 1-isothiocyanato-4-(methylsulfinyl)

Butanesolutionimmediately after puncture when compared with the non-punctured control (Figure 3). The level of degeneration within the $\mathrm{NP}$ and AF were significantly less severe in the treated groups that received 1-isothiocyanato-4(methylsulfinyl) Butane solution after 8 weeks of puncture (Figure 3) $(P<0.05)$ compared with the model saline group. The model saline groups showed a significant high grading score (11.5) among the animals that received phosphatebuffered saline immediately and after 8 weeks of puncture respectively.

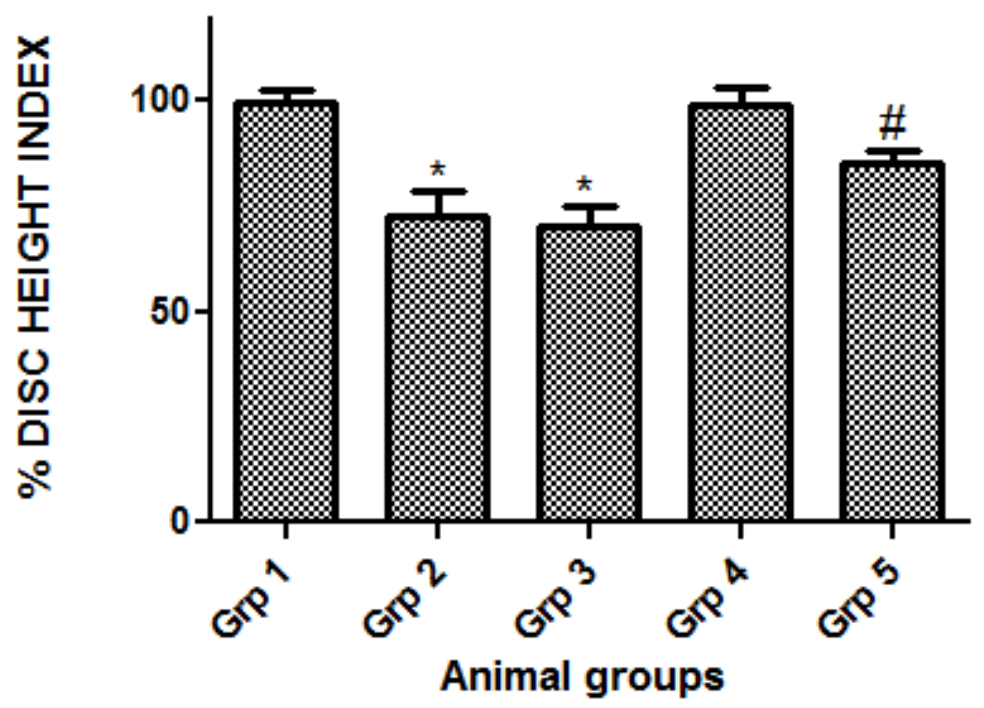

Figure 1: Intervertebral disc height after administration of1isothiocyanato-4-(methylsulfinyl) Butane immediately and 8 weeks after puncture. Values are Mean \pm SEM for 5 rabbits in each group. *: $p<0.05$ as compared to group $1 ; \#: \mathrm{p}<0.05$ as compared to group 1. 

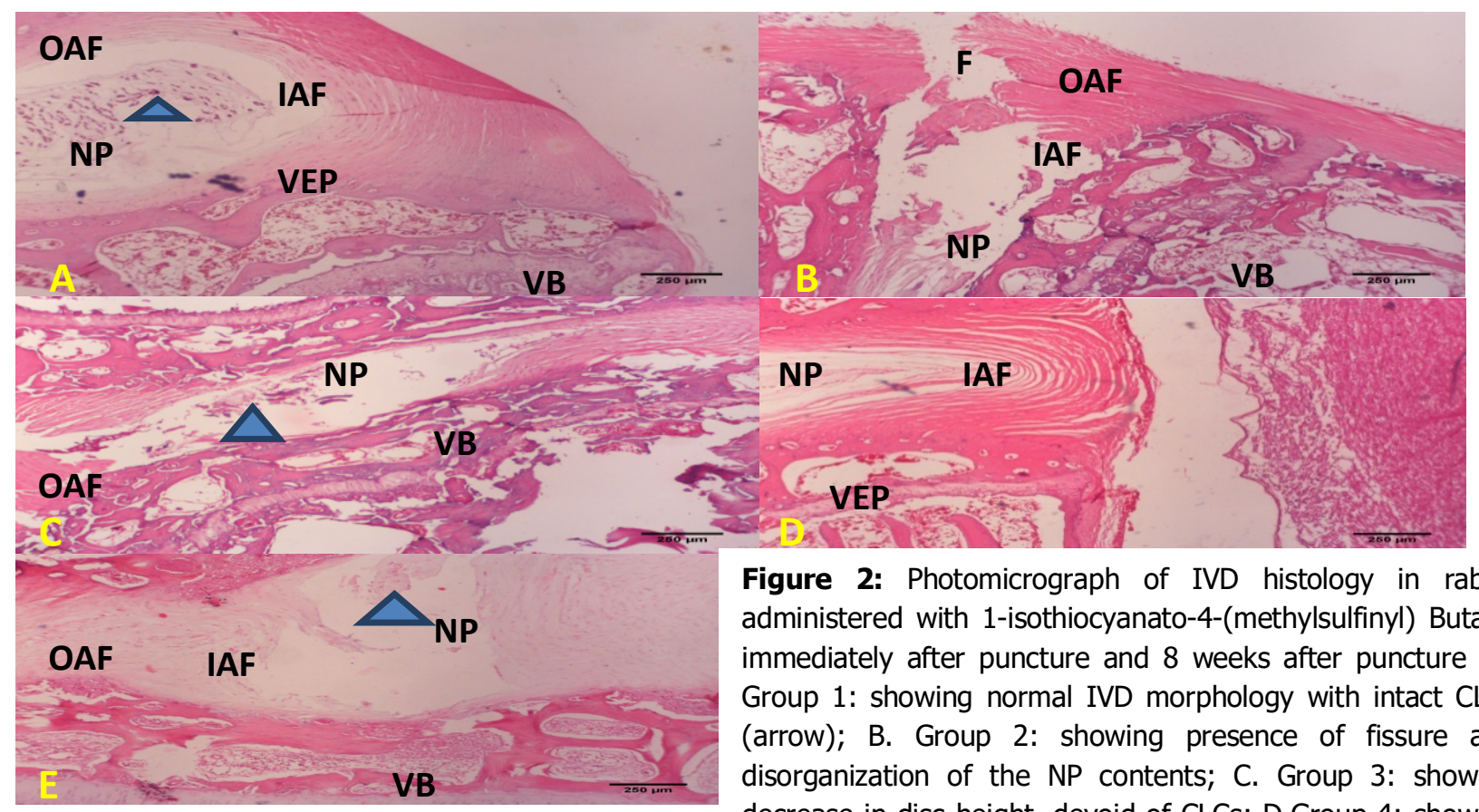

Figure 2: Photomicrograph of IVD histology in rabbit administered with 1-isothiocyanato-4-(methylsulfinyl) Butane immediately after puncture and 8 weeks after puncture (A. Group 1: showing normal IVD morphology with intact CLCs (arrow); B. Group 2: showing presence of fissure and disorganization of the NP contents; C. Group 3: showing decrease in disc height, devoid of CLCs; D.Group 4: showing nearly similar features with group 1; E. Group 5:showing regeneration of NP contents. CLCs: chondrocytes like cells; VB: Vertebral Bone; VEP-Vertebral End Plate; NP- Nucleus pulposus; IAF: Inner Annulus Fibrosus; OAF: Outer Annulus

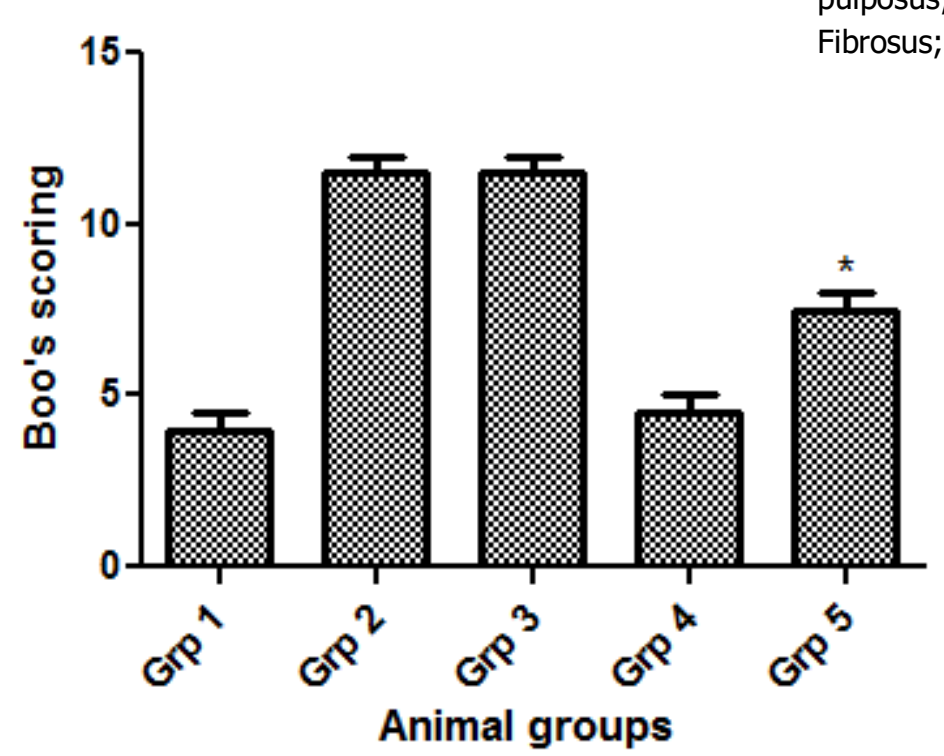

Figure 3: Histological grading scores for level ofdegeneration of rabbit intervertebral disc after 1-isothiocyanato-4(methylsulfinyl) Butane administration. *: $\mathrm{p}<0.05$ as when compared to group 1.

\section{MORPHOMETRY ANALYSIS OF CHONDROCYTE-LIKE CELLS IN IVD.}

Within this present study, one of the major cellular changes was the increasing number of chondrocyte-like cells in the inner AF or in the
NP of the animals administered with1isothiocyanato-4-(methylsulfinyl) Butane. In the NP and inner AF, the number of chondrocyte-like cells in the treated groups across the stages was significantly higher than those of the model 
control groups $(P<0.05)$ (Figure 4), although, no significant difference was found between the model saline groups (Figure 4). In the outer $\mathrm{AF}_{\text {, }}$ no significant differences were found across groups in this present study (Figure 4).

\section{HISTOLOGICAL SECTIONS OF ORGANIZATION OF COLLAGEN FIBRES}

The photomicrograph in group 1 showedoblique concentric direction of collagen fibres, which were continuous and complete within the $\mathrm{AF}$ (Figure 4). The photomicrograph of collagen fibrein the model saline group (group 2) showed disruption in the lamellae structure, partial disorganization in the oblique arrangements of collagen fibrils and loose attachment of the lamellae to the endplate. The treated groups showed nearly similar morphological features in the arrangement of collagen fibres with that of group 1 .The result revealed dense alternating lamellae in the IAF that appears longitudinal in the superficial areas and oblique in the deeper areas. The OAF appears thinner and the fibers were arranged longitudinally. The AF lamellae were firmly attached into the adjacent endplates inform of Sharpey's fibers. Nucleus pulposus showed central area of denser fibrous tissue with numerous rounded chondrocyte like cells.

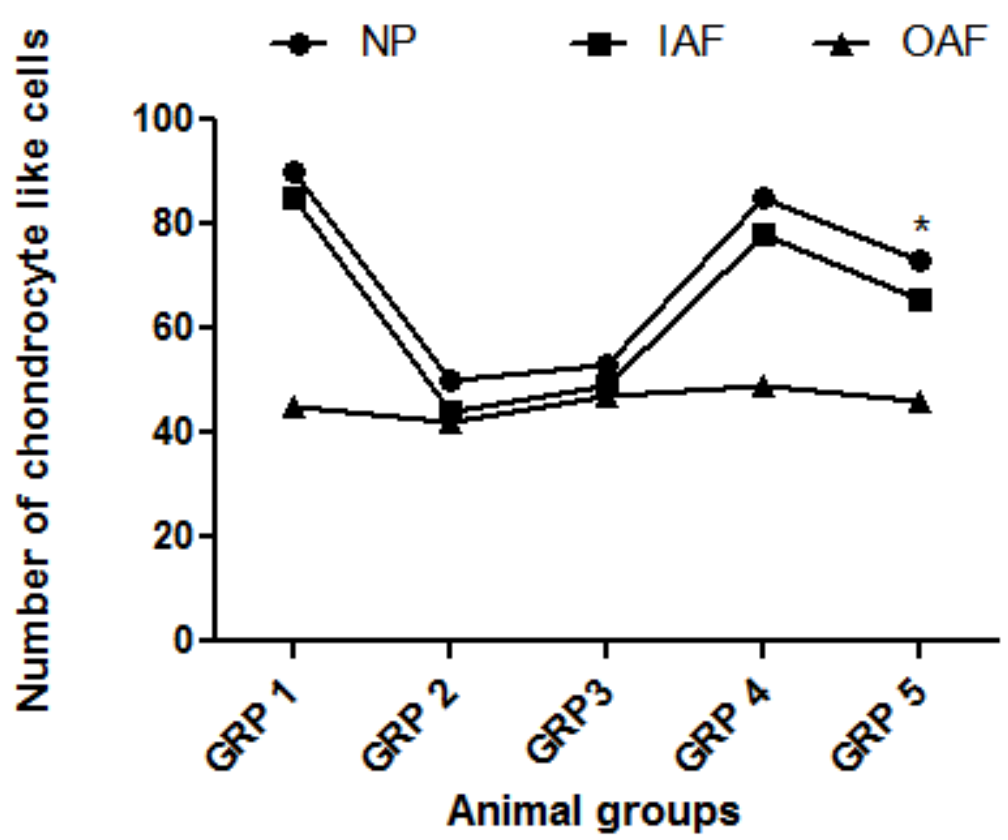

Figure 4:Number of chondrocyte like cells in Nucleus pulposus (NP), Inner Annulus Fibrosus (IAF) and Outer Annulus Fibrosus (OAF) of rabbit intervertebral disc after 1-isothiocyanato-4-(methylsulfinyl) Butane administration. ${ }^{*} \mathrm{p}<0.05$ as compared to group 1.

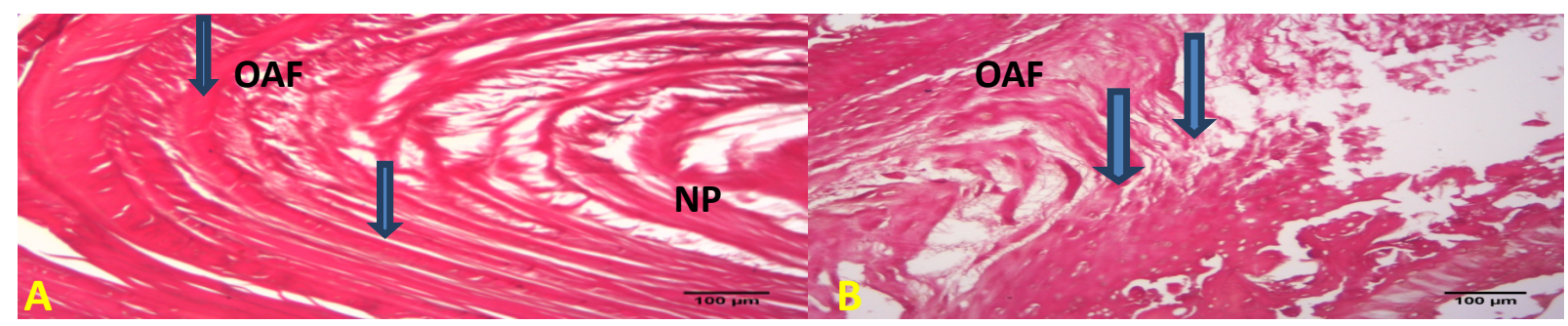




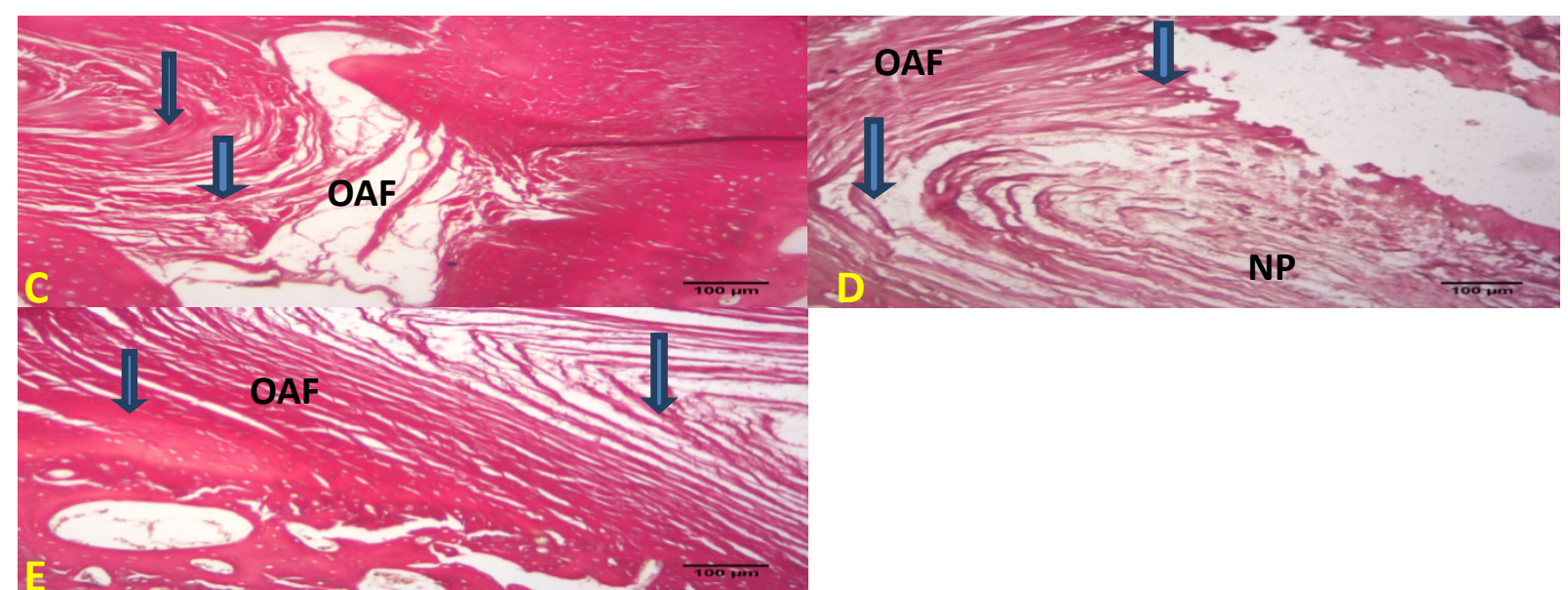

Figure 5: Photomicrograph of IVD collagen fibresin rabbitsadministered with 1-isothiocyanato-4-(methylsulfinyl) Butane immediately after puncture and 8 weeks after puncture (A: Group 1; B: Group 2; C: Group 3; D: Group 4; E: Group 5) showing presence and arrangement of collagen fibres in AF (arrows). VB: Vertebral Bone; VEP-Vertebral End Plate; NP- Nucleus pulposus; IAF: Inner Annulus Fibrosus; OAF: Outer Annulus Fibrosus; H and E: x100.

\section{GENE EXPRESSION LEVELS}

In this study, the relative gene expression levels of aggrecan andCol 2 were decreased $(P<0.05)$, while relative gene expression levels of Col1and MMP 13 increased after annular puncturein the model saline groups (group 2 and 3) (Figure 6; $P<0.05)$ compared with the non-punctured group (group 1). There was no significant difference in the relative gene expression levels of the group that received 1-isothiocyanato-4(methylsulfinyl) Butane compared to the nonpunctured group. However, the expression level of Col 2 and aggrecan were relatively increased compared to model saline groups in response to 1-isothiocyanato-4-(methylsulfinyl) administration $(p<0.05)$.

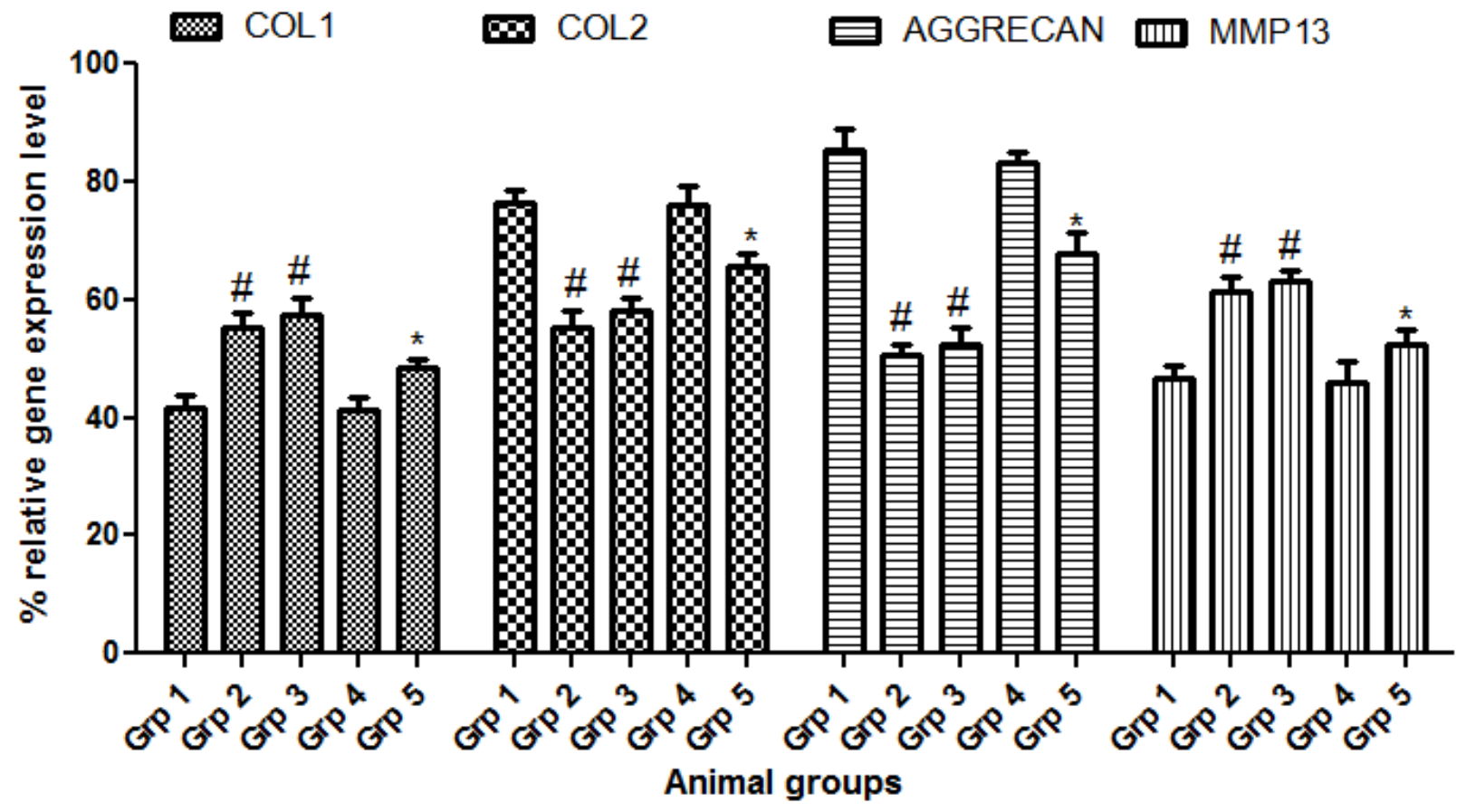

Figure 6: Gene expression level of collagen type 1, collagen type 2, Aggrecan and Matrix Metalloproteinase-13 in rabbits administered with 1-isothiocyanato-4-(methylsulfinyl) Butane immediately and after 8 weeks of puncture, calculated as relative percentages compared with the model 
non-punctured level. ${ }^{*} p<0.05$ as compared to model punctured control; $\# p<0.05$ as compared to model non-punctured control.

IMMUNOHISTOCHEMICAL ANALYSIS.

The immunohistochemical analysis revealed that the expression level of Bax in the model saline groups (group 2 and 3) were higher compared with the non-punctured group (Figure 7) while the treated group that received the administration of 1-isothiocyanato-4(methylsulfinyl) Butane immediately after puncture revealed similar features with that of the non-punctured group (Group 1). However, the expression level of Bax was reduced in the group administered with 1-isothiocyanato-4(methylsulfinyl) Butane after 8 weeks of puncture when compared with model saline group.Quantitative analysis showed that the numbers of positive cells were significantly reduced after the administration of 1isothiocyanato-4-(methylsulfinyl) Butane after 8 weeks of puncture compared with the model saline groups. The administration of 1isothiocyanato-4-(methylsulfinyl)

Butaneimmediately after puncture revealed no significant difference in the number of positive cells when compared withgroup $1 \quad(P<0.05)$ (Figure 7).

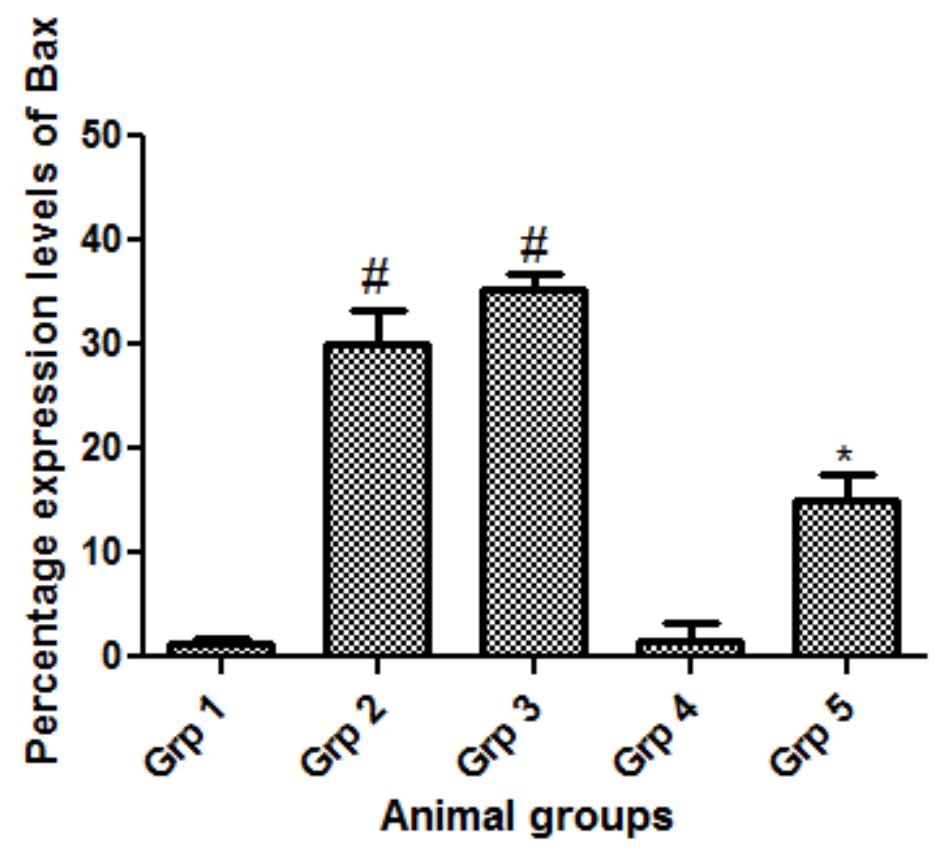

Figure 7: Statistical analysis ofBax protein expression levels. $* P<0.05$ compared with groups 2 and 3; $\# P<0.05$ compared with non-puncture model group (group 1 ).

\section{DISCUSSION}

Therapeutic strategies currently used for patients with disc degeneration remain symptomatic - dedicated mainly to relieving painful symptoms of low back pain. The pain experienced was as a result of the imbalance between anabolism and catabolism which contributes to the disorganisation of the extracellular matrix(Christian et al., 2001).
The age of the rabbitused for this study ranged between 20-24 weeks with an average weight of $3.22 \mathrm{~kg}$. This is to ascertain that no degenerative change occurred in the animalssince no morphological evidence of degenerative changes of the intervertebral disc was observed in previous study till 24 weeks age in rabbit(Kim et al., 2005). 
This study examined the efficacy of the oral administration of 1-isothiocyanato-4(methylsulfinyl) Butane into the well-established rabbit annular needle puncture model of disc degeneration. The results of this study demonstrated that the administration of 1isothiocyanato-4-(methylsulfinyl) Butane was effective in preventing and restoring disc height in this animal model.

In this study, the annular punctured model exhibited slow progressive disc degeneration as evident by radiology (in the percentage $\mathrm{DHI}$ ) (Table 2) and histology (in the histological score) (Figure 4)beginning at 2 weeks till at least 8 weeks post-surgery (although there were no significant differences in histological grades between baseline and 2 weeks post-surgery). Thus, this annular puncture model revealed a progressive disc degeneration overtime resembling human intervertebral disc degeneration (IDD) (Masuda et al., 2005; Freemont, 2009). The disruption in the cytoarchitecture within the annulus fibrosus in several animal models and in humans has revealed the occurrence of disc degeneration (Olsewskiet al., 1996; Carrageeet al.,2009).The significant correlations between \%DHI values and histological scores (Figure 7) could affirm that DHI can be used as a good indicator in determining the level of disc degeneration (Masuda et al., 2005).

The present study showed that the administration of 1-isothiocyanato-4(methylsulfinyl) Butane immediately after annular puncture prevented the progression of IVDD as evident in the \%DH (Figure 1). Moreover, there were no apparent side effects such as ossification in the IVD and anatomical or physiological changes in NP cells in this study. The present study revealed various histological alterations, ranging from discrete disruption of the nucleus pulposus, decrease nucleus pulposus cells and decrease lamella disorganisation and complete obliteration of its cavity. The histological analysis revealed a reparative effect of the

administration of 1-isothiocyanato-4(methylsulfinyl) Butane on the degenerated IVD.
Histological observation of the IVD sections of the model control group revealed notable progression in degeneration in which most of the NP contents have been lost and collapsed, wavy fibrocartilage lamella and associated fibrochondrocyte-like cells of the AF which was restored after the administration of 1isothiocyanato-4-(methylsulfinyl).In all of the experimental groups, the invasion of blood vessels or inflammatory cells was not observed within the discs. Furthermore, no ossification of exclusively IVD tissues was found in any of the samples, although there was significant osteophyte formation at the edge of the vertebral body.

The general morphology of the disc showed a centrally located NP surrounded by AF anchoring to EP. Within the AF, the arrangement of fibers in the outer (posterior) lamellae that continues with the longitudinal ligament and vertebral bodies facilitates the discs movement and flexibility. It was evident that collagen fibers were more in inner $\mathrm{AF}$ than outer $\mathrm{AF}$ throughout the groups administered with the 1isothiocyanato-4-(methylsulfinyl) Butane. The elastic fibres were less in outer AF than in inner AF across the groups. The difference between the collagen and elastic components of the disc could pose a high risk factor in the etiopathogenesis of disc degeneration and acts as a precursor for degenerative changes thereby causing LBP (Choudharyet al., 2011). Structural differences in the outer and inner AF also affects the strength of the disc, in which the outer AF is weaker and more prone to damage and this is in correlation with clinical observations whereby most of the assault within the IVD occurs in the outer AF (Battie 2006; Misterskaet al., 2011; Wang et al., 2012).

The elastic fibers are organized within the NP with long straight fibres radially oriented and anchored perpendicularly or obliquely into the EP. Within the AF, elastic fibers appear densely distributedin thke region between the lamellae and in bridges across the lamellae. Variation in structural appearance of elastic fibers depends on functional requirement. In the nuchal and other ligaments, they appear rod-like, concentric 
in blood vessels and three- dimensional meshwork in auricular cartilage. Elastic fibres are predominantly found in tissues that require resilience and elasticity for their function (Cleary and Gibson, 1996). The morphology of AF cells ranges from 'chondrocyte-like' in the inner AF to fibroblast-like in the outer AF, while morphology of NP cells are more typically chondrocyte-like (Buckwalter, 1995; Urban \& Roberts, 2003). This morphological attribute correlates with a change in collagen production from type II collagen in the NP to type I collagen in the outer AF (Buckwalter, 1995; Urban \& Roberts, 2003).

Structural disorganization of the disc tissue is ultimately due to alteration in the NP cell phenotype of the expression levels of molecules in the onset of disc degeneration (COL1, COL2, AGC and MMP13) (Sakai et al., 2005; Tomoyasuet al., 2007; Alsousou et al., 2012).

The results shows an increase in COL2 and aggrecan expression with a concomitant decrease in COL1 and MMP13 in the treated groups when compared to the model group suggesting that NP cells experience a process of dedifferentiation. Interestingly, this dedifferentiation process has been well described in cultured articular chondrocytes (Schnabel et al., 2002) and in osteoarthritic joints (Clouet et al., 2009). The result also suggested that during the process of IVD aging, the molecules with a longer half-life (collagen type II) exhibit an early decrease in the corresponding transcript levels. Conversely, the molecules with a shorter half-life (aggrecan) have a transcript expression levels that start to decline later on. Among the various genes modulated in osteoarthritic chondrocytes (Sakai et al., 2005), MMP-13 is known to degrade collagens and glycosaminoglycans (Roberts et al., 2000). The increase in MMP13 could therefore be a major contributor of IVD degeneration (Alsousouet al., 2012) as it has been extensively reported in cartilage degradation during osteoarthritis (Sakai et al., 2005).
In this study, the expression levels of Bax were increased in the model control group that underwent annular puncture when compared to the non-puncture positive control group (group 1). In addition, the expression levels of Bax among the groups administered with 1isothiocyanato-4-(methylsulfinyl) Butane were reduced when compared with group 2. It could be deduced that Bax was involved in the process of nucleus pulposus cell apoptosis, thereby affecting the anabolic and catabolic mechanism between cell proliferation and apoptosis; ultimately resulting to degenerated disc (Wang et al., 2008; Wang et al., 2013).

Previous research revealed that traumatic injury induced a caspase cascade response in affected disc thereby upregulating the expression level of proapoptotic protein (Bax) and ultimately leading to cell apoptosis (Yang et al., 2016).

The inhibition of caspase proteins was deduced to be the underlying mechanism through whichBax, a proapoptotic gene, promotes caspase activity (Yang et al., 2016). The relationships between Bax and other antiapoptotic proteins could be used for the estimation of apoptosis cell sensitivity (Gruber et al., 1998).

\section{Conclusion}

The present study has provided radiographic and morphological evidence that disc degeneration occurs in annular puncture model of rabbit intervertebral disc. The results showed induced initiation and progression of disc degeneration from biological, biochemical and morphologic perspectives. Furthermore, the administration of 1-isothiocyanato-4-(methylsulfinyl) Butane was effective in preventing and restoring disc height and increasing chondrocytic cells in the intervertebral disc of the rabbit model. The present study also demonstrated that 1isothiocyanato-4-(methylsulfinyl) Butane had anabolic effects on degenerated disc and could provide valuable information for consideration in clinical trials.

COMPETING INTERESTS: Authors have declared that no competing interests exist.

\section{REFERENCES}

1. Alsousou J, Ali A, Willett K, Harrison P. 2012. The role of platelet-rich plasma in tissue regeneration. Platelet,24 (3): 173-182. 
2. Battie MC, Videman T. 2006. Lumbar disc degeneration: epidemiology and genetics. Journal of Bone \& Joint Surgery, 88 (2): 3-9.

3. Boos N, Weissbach S, Rohrbach $H$, Weiler C, Spratt KF, Nerlich AG. 2002. Classification of agerelated changes in lumbar intervertebral discs. Spine,27: 2631-2644.

4. Buckwalter JA. 1995. Ageing and degeneration of the human intervertebral disc. Spine, 20: 13071314.

5. Carragee EJ, Don AS, Hurwitz EL, Cuellar JM, Carrino JA, Herzog R. 2009. Does discography cause accelerated progression of degeneration changes in the lumbar disc: a ten-year matched cohort study. Spine, 34(8): 2338-2345.

6. Childs J, Fritz J, Flynn T, Irgang J, Johnson K, Majkowski G, Delitto A. 2004. A clinical predication rule to identify patients with low backpain most likely to benefit from spinal manipulation: A validationstudy. Annals of Internal Medicine, 141(12): 920-928.

7. Choudhary S, Sharma AK, Singh H, Richa S, Sharma N. 2011. Light microscopic study of the lumbar intervertebral disc showing inherent difference between anterior \& posterior annulus fibrosus-A risk factor for posterior disc herniation. Journal of Korean Science, 13: 89-91.0

8. Christian WA, Metzdorf A, Hodler J,Boos N. 2001.Spine, 26(17): 1873-1878.

9. Cleary EG, Gibson MA. 1996. Extracellular Matrix. Harwood Academic Publishers, Amsterdam. vol. 2 (Comper, W. D., ed.), 95- 140.

10. Clouet J, Vinatier C, Merceron C. 2009. From osteoarthritis treatments to future regenerative therapies for cartilage. Drug Discovery, 14(19): 913-925.

11. Dinkova-Kostova AT. 2013. Chemoprotection against Cancer by Isothiocyanates: A Focus on the Animal Models and the Protective Mechanisms. Topics in Current Chemistry, 329: 179-201.

12. Egner PA, Chen JG, Zarth AT, Ng DK, et al. 2014. Rapid and Sustainable Detoxication of Airborne Pollutants by Broccoli Sprout Beverage: Results of a Randomized Clinical Trial in China. Cancer Prevention Research (Philadelphia), 7: 813-823.

13. Elliott DM, Sarver JJ. 2004. Young investigator award winner: validation of the mouse and rat disc as mechanical models of the human lumbar disc. Spine, 29:713-722.

14. Freemont AJ. 2009. The cellular pathobiology of the degenerate intervertebral disc and discogenic back pain. Rheumatology 48, 5-10.

15. Gao X, Talalay P. 2004. Induction of Phase 2 Genes by Sulforaphane Protects Retinal Pigment Epithelial Cells against Photooxidative Damage. Proceedings of National Academy Sciences of the United States of America, 101: 10446-10451.

16. Gaona-Gaona L, Molina-Jijón E, Tapia E et al. J. 2011. Protective Effect of Sulforaphane Pretreatment against Cisplatin-Induced Liver and Mitochondrial Oxidant Damage in Rats. Toxicology, 286: 20-27.

17. Gruber HE, Johnson T, Norton HJ, Hanley EN. 2002. The sand rat model for disc degeneration: radiologic characterization of age-related changes: cross-sectional and prospective analyses. Spine, 27: 230-234

18. Kensler TW, Chen JG, Egner PA et al. 2005. Effects of Glucosinolate-Rich Broccoli Sprouts on Urinary Levels of Aflatoxin-DNA Adducts and PhenanthreneTetraols in a Randomized Clinical Trial in HeZuo Township, Qidong, People's Republic of China. Cancer Epidemiology, Biomarkers \& Prevention, 14: 2605-2613.

19. Kensler TW, Egner PA, Agyeman AS, Visvanathan K et al. 2013. Keap1-nrf2 Signaling: A Target for Cancer Prevention by Sulforaphane. Topics in Current Chemistry, 329: 163-177.

20. Le Maitre CL, Freemont AJ, Hoyland JA. 2007. The role of interleukin-1 in the pathogenesis of human intervertebral disc degeneration. Arthritis Research Therapy,7: 732-745.

21. Lenzi M, Fimognari C, Hrelia P. 2014. Sulforaphane as a Promising Molecule for Fighting Cancer. Cancer Treatment and Research, 159: 207-223. 
22. Lopez A, Mathers C, Ezzati M, Jamison D, Murray J. 2006.Global and regional burden of disease and risk factors, 2001: Systematic analysis of population health data. Lancet, 367:1747-1757.

23. Lotz JC. 2004. Animal models of intervertebral disc degeneration: lessons learned. Spine, 29: 2742-2750

24. Masuda K, Aota Y, Muehleman C, Imai Y, Okuma M, Thonar EJ, Andersson GB, AnHS. 2005. A novel rabbit model of mild, reproducible disc degeneration by an annulus needle puncture: correlation between the degree of disc injury and radiological and histological appearances of disc degeneration. Spine,30: 5-14.

25. Masuda K, Imai Y, Okuma M, Muehleman C, Nakagawa K, Akeda K, Thonar E, Andersson G, An HS. 2006.Osteogenic protein-1 injection into a degenerated disc induces the restoration of disc height and structural changes in the rabbit annular puncture model. Spine,30: 742-754.

26. Misterska E, Jankowski R, Glowacki M. 2011. Quebec Back Pain Disability Scale, Low Back Outcome Score and Revised Oswestry low back pain disability scale for patients with low back pain due to degenerative disc disease: evaluation of Polish versions. Spine, 3(6): 1722-1729.

27. Murray J, Lopez A. 1997. Global mortality, disability, and the contribution of risk factors: Global Burden of Disease Study. Lancet, 349: 1436-1442.

28. Olsewski JM, Schendel MJ, Wallace L, Ogilvie JW, Gundry CR. 1996. Magnetic resonance imaging and biological changes in injured intervertebral discs under normal and increased mechanical demands. Spine, 2(1): 1945-1951.

29. PodichettyVK(2007). The aging spine: the role of inflammatory mediators in intervertebral disc degeneration. Cell and Molecular Biology,5(3): 4-18.

30. Rousseau MA, Ulrich JA, Bass EC, Rodriguez AG, Liu JJ, Lotz JC. 2007. Stab incision for inducing intervertebral disc degeneration in the rat. Spine, 32: 17-24

31. Sakai D, Mochida J, Iwashina T, Watanabe T, Nakai T, Ando K, Hotta T. 2005. Differentiation of mesenchymal stem cells transplanted to a rabbit degenerative disc model: potential and limitations for stem cell therapy in disc regeneration. Spine, 30: 2379-2387.

32. Schnabe M, Marlovits S,Eckhoff G. 2002. Dedifferentiation-associated changes in morphology and gene expression in primary human articular chondrocytes in cell culture. Osteoarthritis Cartilage, 10(1): $62-70$.

33. Shirai Y, Fujita Y, Hashimoto K. 2012. Effects of the Antioxidant Sulforaphane on Hyperlocomotion and Prepulse Inhibition Deficits in Mice after Phencyclidine Administration. Clinical Psychopharmacology and Neuroscience, 10: 94-98.

34. Shirai Y, Fujita Y, Hashimoto R, Ohi K, Yamamori H, et al. 2015. Dietary Intake of SulforaphaneRich Broccoli Sprout Extracts during Juvenile and Adolescence Can Prevent Phencyclidine-Induced Cognitive Deficits at Adulthood. PLoS One, 10: 127244.

35. Talalay P, Fahey JW, Holtzclaw WD, Prestera T, Zhang Y. 1995. Chemoprotection against Cancer by Phase 2 Enzyme Induction. Toxicology Letters, 82-83: 173-179.

36. Tomoyasu A, Higashio K, Kanomata K, Goto M, Kodaira K, Serizawa H. 2007. Platelet-rich plasma stimulates osteoblastic differentiation in the presence of BMPs. Biochemical and Biophysical Research Communication, 361: 62-67.

37. Toyama T, Shinkai Y, Yasutake A, Uchida K, Yamamoto M, Kumagai Y. 2011. Isothiocyanates Reduce Mercury Accumulation via an Nrf2-Dependent Mechanism during Exposure of Mice to Methylmercury. EnvironmentalHealth Perspectives, 119: 1117-1122.

38. Urban JP, Roberts S. 2003. Degeneration of the intervertebral disc. Arthritis Research Therapy, 5: $120-130$.

39. Ushida Y, Talalay P. 2013.Sulforaphane Accelerates Acetaldehyde Metabolism by Inducing Aldehyde Dehydrogenases: Relevance to Ethanol Intolerance. Alcohol and Alcoholism, 48: 526534. 
40. Vialle EN, Vialle LR, G,Arruda OA. 2012.Histomorphometric analysis of experimental disc degeneration. Global Spine Journal,2: 129-136.

41. Wang SL, Yu YL, Tang CL, Lv FZ. 2013. Effects of TGF-beta 1 and IL-1-beta on expression of ADAMTS enzymes and TIMP-3 in human intervertebral disc degeneration. Experiment and Therapeutic Medicine, 6: 1522-1526.

42. Wang Y, Chen HB, Zhang L, Zhang LY, Liu JC, Wang ZG. 2012. Influence of degenerative changes of intervertebral disc on its material properties and pathology. Chinese Journal of Traumatology, 1(5): 67-76.

43. Wang Y, Chen HB, Zhang L, Zhang LY, Liu JC, Wang ZG. 2008. Influence of degenerative changes of intervertebral disc on its material properties and pathology. Chin J Traumatology,1(5): 67-76.

44. Woolf A, Pfleger B. 2003.Burden of major musculoskeletal conditions. Bu II World Health Organ, 81(9): 646-656.

45. Yang J, Liu X, Bhalla K, Kim CN, Ibrado AM, Cai J. 2016. Prevention of apoptosis by Bc1-2: Release of cytochrome $c$ from mitochondria blocked. Science, 27(5): 1129-1132.

46. Young-Joon K. 2013. A minimally invasive Rabbit Model of progressive and reproducible Disc Degeneration confirmed by Radiology, Gene Expression and Histology. Journal of Korean Neurosurgical Society, 5(3): 323-330.

47. Zhang Y, Talalay P, Cho CG, Posner GH. 1992. A Major Inducer of Anticarcinogenic Protective Enzymes from Broccoli: Isolation and Elucidation of Structure. Proceedings of the National Academy of Sciences of the United States of America, 89: 2399-2403.

48. Zhao Z, Liao G, Zhou Q, Lv D, Holthfer H, Zou H. 2016.Sulforaphane attenuates contrast-induced nephropathy in rats via Nrf2/HO-1 pathway. Oxidative Medical Cell Longevity, (9)8: 25-32. 